\title{
Myocardial involvement in infectious hepatitis
}

\author{
N. Nagaratnam \\ M.D., M.R.C.P.(Glasg.)
}

\author{
D. P. K. M. De Silva \\ D.C.P.(Lond.), D.Path., M.R.C.Path.(Lond.)
}

Physician

\author{
K. R. W. Gunawardene \\ M.B.B.S.(Cey.)
}

House Physician

Government Hospital, Gampaha, W. P., Ceylon

\begin{abstract}
Summary
Four cases with myocarditis due to infectious hepatitis are described. Infectious hepatitis, though it predominantly affects the liver could be a disseminated illness and myocarditis, usually benign, could be a serious complication as in one of the cases in this series.
\end{abstract}

A WIDE variety of infectious diseases may affect the heart giving rise to myocarditis. During life the diagnosis often depends upon the recognition of the possibilities of myocarditis in the particular infection. Viruses, bacteria and parasites have all been incriminated as a cause of this disorder. There have been very few reports in the literature on infectious hepatitis causing myocarditis.

This paper presents four cases of myocarditis due to infectious hepatitis. They have been described in detail in another communication (Nagaratnam, Gunawardena \& De Silva, 1971). Only the myocardial aspects are described here.

\section{Case reports}

\section{Case 1}

RAS (28155), a male, aged 51 years, was admitted on 28 October, 1970, with a history of jaundice of 20 days' duration. He took alcohol occasionally. On admission, he complained of some discomfort in his chest.

Examination revealed a deeply jaundiced individual. His blood pressure was $130 / 80 \mathrm{mmHg}$. There were no bruits or additional heart sounds. The lungs were clear. The liver was enlarged two fingerbreadths below the right costal margin. There was ascites and slight oedema of the legs. The results of the liver function tests are shown in Table 1. An electrocardiogram showed low $\mathrm{T}$ waves in II, inversion of the T waves in III, aVF, V1-V6 on 28 October, 1970 (Fig. 1) and 4 days later showed upright $\mathrm{T}$ waves.

Comment. The clinical and biochemical findings were those of infectious hepatitis. Clinically there were no signs related to the cardiovascular system.

TABLE 1. Summary of biochemical and haematological findings

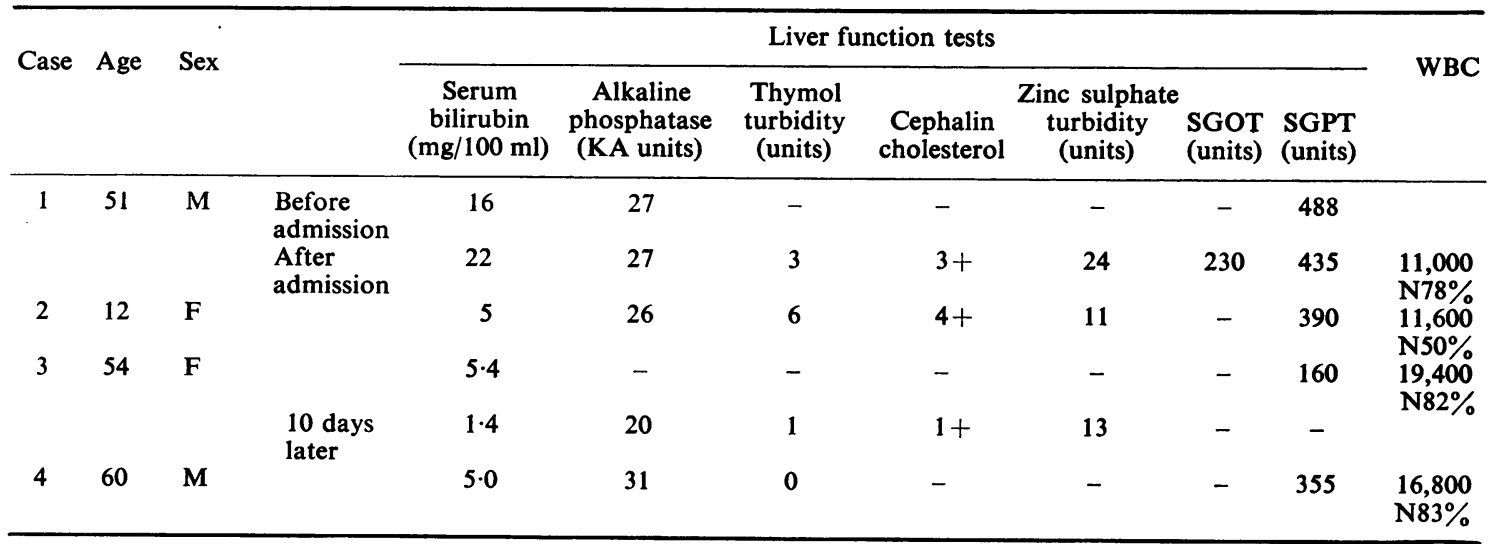




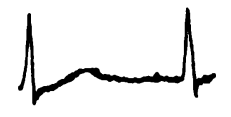

I

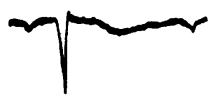

aVR

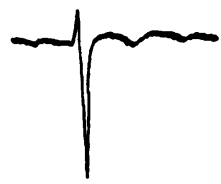

V2
aVL

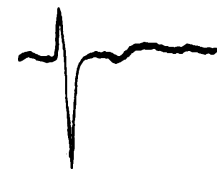

V4
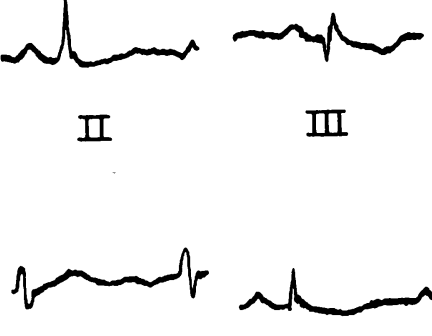

III

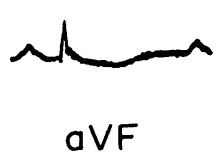

FIG. 1. Electrocardiogram of Case 1 showing nonspecific $T$ wave changes.

The electrocardiogram showed transient non-specific $T$ wave changes.

\section{Case 2}

A (21952), a school-girl, aged 12 years, was referred from her hospital with a history of fever, restlessness and a generalized convulsion. She had been delirious that evening. She had a contact history with a case of infectious hepatitis.

On examination, she had no icterus but became jaundiced the following day.

Results of the liver function tests are summarized in Table 1.

Progress. On the twelth day, she became suddenly breathless and her pulse was rapid and irregular. Her lungs were clear. Other than a tachycardia there were no murmurs or abnormal heart sounds.

The electrocardiogram revealed sinus tachycardia (rate 140/min), isoelectric T waves in II and inverted T waves in III, aVF and V2-V6. She was treated with bed rest and digitalis. Her condition gradually improved but her pulse remained irregular. An electrocardiogram done 6 days later showed a slower rate but the changes remained as in the earlier record. $\mathrm{X}$-ray of the chest before discharge from hospital showed a normal cardiac silhouette with minimal cardiac enlargement. The lung fields were clear.

Comment. This young girl had infectious hepatitis with neurological complications at the onset of her illness. She developed myocarditis on the twelfth day of her illness from which she recovered without any sequelae.

\section{Case 3}

KGN.CN (13641, 17429), a female, aged 54 years, was referred from her local hospital with fever, jaundice and hypotension of 4 days' duration.

On admission, she complained of pain in chest and breathlessness. She was jaundiced.

Examination of the cardiovascular system revealed a blood pressure of $65 / 50 \mathrm{mmHg}$. The jugular venous pressure was not elevated. Both sounds were heard at the apex. There was a short systolic murmur at the apex and a triple rhythm. The liver was not palpable.

Results of the liver function tests are shown in Table 1.

Progress. With treatment the blood pressure improved and an electrocardiogram done on the fifth day showed low voltage complexes with slurred QRS complexes in II, III, aVF with low and flattened T waves. She made an uneventful recovery.

She was re-admitted on 9 July, 1970, 6 weeks later, with fever and a haemolytic anaemia.

Comment. This lady undoubtedly had infectious hepatitis. She had hypotension at the time of her admission and the electrocardiogram showed myocardial involvement.

\section{Case 4}

EA (25859), a cultivator, aged 60 years, was admitted with a history of jaundice of 1 week' duration and weakness and paraesthesiae of the lower limbs.

Examination revealed a jaundiced individual whơ was drowsy. His liver was not palpable. He was treated as a case of impending hepatic coma due to infectious hepatitis. The liver function tests are shown in Table 1.

Progress. Five days later, he became pale and breathless and the findings were consistent with a haemolytic episode. He complained of pain in chest and his condition gradually deteriorated with increasing breathlessness and he developed oedema of his lower extremities. An electrocardiogram done 2 days prior to his death (on the sixteeth day of his illness) revealed irregularity in the rhythm with ventricular extrasystoles and $\mathrm{T}$ wave inversion in II, III and aVF (Fig. 2).

Necropsy. There were $2 \mathrm{oz}$ of yellow pericardial fluid. The heart was pale and flabby and was stained yellow. There was no abnormality of the valves, aorta and pulmonary arteries. The coronary arteries $N$ were normal. Microscopic appearances: sections of the myocardium showed small foci of degenerative $N$ changes, necrosis of muscle fibres and interstitial $\underset{\omega}{N}$ oedema (Fig. 3). There were focal collections of inflammatory cells, mainly mononuclears with a few polymorphs. The adjacent endocardium also showed cellular infiltration (Fig. 4). The areas of necrosis $\stackrel{?}{+}$ 


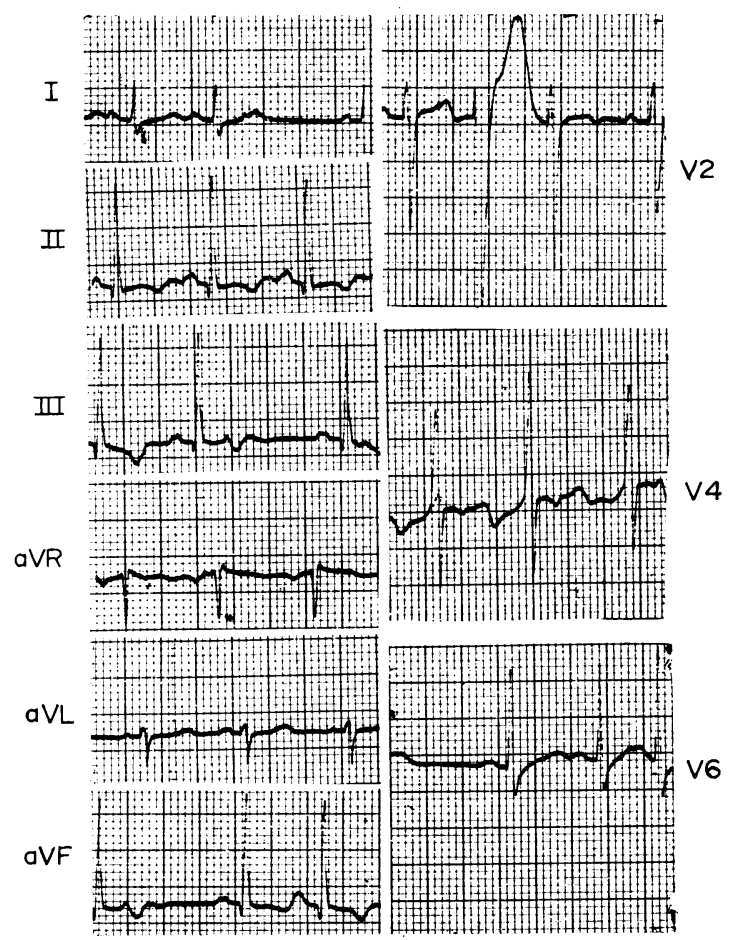

FIG. 2. Electrocardiogram of Case 4 showing irregularity in rhythm with ventricular ectopics and $T$ wave changes.

were marked in the right ventricle near the interventricular septum. No haemorrhages were seen in the bundle of His. The changes in the liver were consistent with that seen in infectious hepatitis.

Comment. The laboratory and necropsy liver findings were those seen in infectious hepatitis. On the twelfth day of illness he developed an acute haemolytic anaemia and subsequently developed cardiac failure. The electrocardiogram and the histological changes were those of myocarditis. Death could have been due to both the haemolytic and the myocardial complications.

\section{Discussion}

Infectious hepatitis is considered a generalized disease and its manifestations in tissues other than the liver has been studied by several workers (Condrad, Schwartz \& Young, 1964). Myocarditis has been demonstrated in necropsy material of patients dying of fulminant hepatitis (Saphir, Amromin \& Yokoo, 1956).

It is important to recognize that the heart can be affected in this illness for not only is it a widespread disease with an increasing incidence in many countries, but also that this complication can be potentially a serious one.

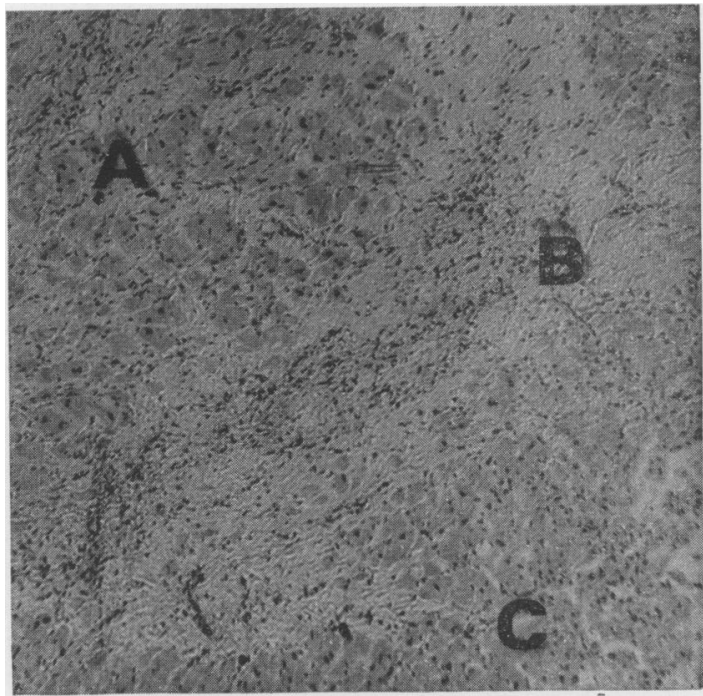

Fig. 3. Case 4. Section of the myocardium showing necrosis and degenerative changes of the muscle fibres (A), interstitial oedema and cellular infiltrate (B). The surviving muscle fibres show hyperchromatic nuclei (C). H. and E. $\times 72$.

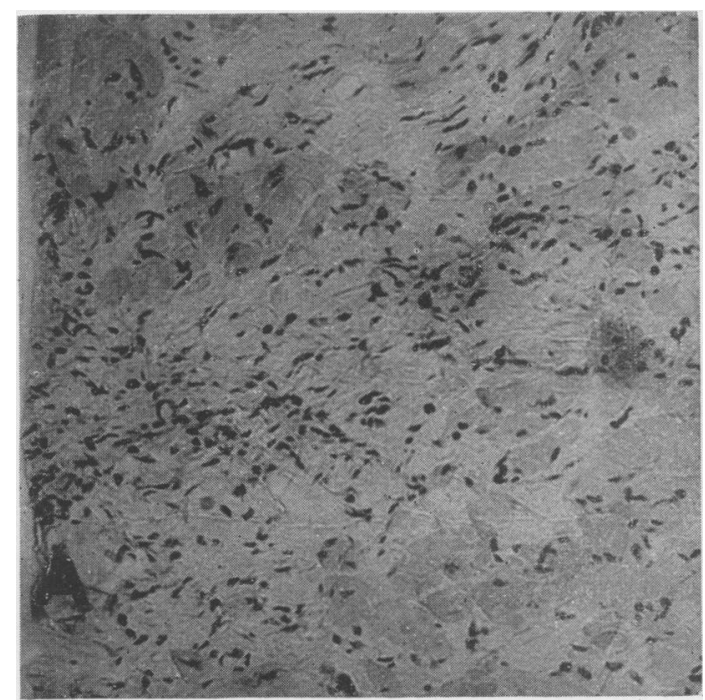

FIg. 4. Case 4. Section of the myocardium showing degenerative changes and necrosis of muscle fibres, interstitial oedema and also involvement of the endocardium (A). H. and E. $\times 218$.

The clinical features in myocarditis can be so variable that it can often be missed. It may be minimal, limited to subtle electrocardiographic changes (Case 1) or to the development of extra- 
systoles (Case 2) and these may be the only findings suggesting that the heart is involved. On the other hand, it can be so glaring as the developed congestive cardiac failure with cardiac enlargement, gallop rhythm and death (Case 4) or hypotension and peripheral circulatory failure (Case 3 ).

The four patients in this study group had jaundice and the clinical and biochemical findings were consistent with that of infectious hepatitis.

Hepatitis with jaundice occurs in about $10 \%$ of all patients with infectious mononucleosis and myocarditis is a known complication. In some instances it may be clinically indistinguishable from infectious hepatitis but the characteristic clinical and haematological findings of infectious mononucleosis were not present in our cases. There is a difference of opinion concerning the exact nature of the hepatic lesion in infectious mononucleosis and it may be indistinguishable from that seen in infectious hepatitis. The findings in the single case where liver tissue was available showed parenchymal cell damage and the presence of lipochrome-containing Kupffer cells which are in favour of a diagnosis of infectious hepatitis (Sullivan et al., 1957). Furthermore, infectious hepatitis is endemic in this country.

Wood (1946) described definite myocarditis in one of his ten cases with infectious hepatitis. Saphir et al. (1956) in a study of clinical records and necropsy findings found four instances of virus hepatitis with myocarditis. According to them the main characteristic findings were minute foci of necrosis of isolated muscle bundles surrounded by cells. A more diffuse myocarditis was sometimes found characterized by oedematous fluid with few interspersed inflammatory cells. These were the findings in Case 4요 in this series. In three of their cases there was involve- $c$ ment of the bundle of His with necrosis, haemorrhage $\Rightarrow$ and acute inflammatory exudate. No changes weres? seen in the bundle of His in Case 4.

The mechanism of heart disease is not clearly흠 understood. The virus may directly damage the $\frac{\bar{\omega}}{\bar{\phi}}$ tissues or may give rise to a hypersensitivity or auto- $\mathbb{\Phi}$ immune reaction. This usually occurs on or abouto the seventh to tenth day after the initial virus. infection. Three of the four cases of Saphir et al. (1956) had a short clinical course and the other died $\vec{\omega}$ on the twenty-first day of illness. In one of our cases the onset of myocarditis was as early as the fourtho day of illness and in the others in the second and 3 . third week.

\section{References}

Condrad, M.E., Schwartz, F.D. \& Young, A.A. (1964)ळ Viral hepatitis-a generalized disease. A study of renal ${ }^{\mathcal{G}}$ gastro-intestinal and haematological abnormalities. Amexi음 can Journal of Medicine, 37, 789.

Nagaratnam, N., Gunawardena, K.R.W. \& De Silka D.P.K.M. (1971) Infectious hepatitis-a multi-systên disease. (In preparation.)

SAPHIR, O., Amromin, G.D. \& Yokoo, H. (1956) Myocardiüs in viral (epidemic) hepatitis. American Journal of Medicgl@ Science, 231, 168.

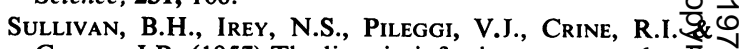
GiBSON, J.R. (1957) The liver in infectious mononucleos: American Journal of Digestive Diseases, 2, 210.

Wood, D.A. (1946) Quoted by Saphir et al. (1956). 